In Proc. IEEE Int. Conf. Image Process., Austin, TX, 1994, vol. 1, pp. 288-292.

\title{
EDGE DETECTION IN ECHOCARDIOGRAPHIC IMAGE SEQUENCES BY 3-D MULTISCALE ANALYSIS
}

\author{
Iztok Koren, Andrew F. Laine, Jian Fan, and Fred J. Taylor \\ Computer and Information Sciences Department \\ University of Florida, Gainesville, FL 32611-2024 \\ Email: Laine@cis.ufl.edu
}

\begin{abstract}
Robust and reliable edge detection is an important step for accomplishing automatic detection of heart wall boundaries in echocardiograms. We present an edge detection algorithm that makes use of both spatial and temporal information. Our algorithm is comprised of (1) a 3-D discrete dyadic wavelet transform carried out on a sequence of images, (2) edge detection that is carried out by maxima detection and a search strategy for maxima curves within the transform space.

Using detected edges and "a priori" knowledge of cardiographic features we demonstrate the performance of fully automatic detection of epicardial and endocardial boundaries along the posterior and anterior walls in 2-D short-axis echocardiographic image sequences.
\end{abstract}

\section{INTRODUCTION}

Two-dimensional (2-D) echocardiography is a noninvasive ultrasonic technique widely used to characterize cardiac function. Ultrasound imaging is more attractive, convenient, and cost less than nuclear or X-ray radiation modalities. Poor quality of ultrasound images, however, makes automatic processing difficult. Low contrast, dropouts, blur, and distortions are some of the problems frequently encountered in ultrasound images.

A sequence of echocardiographic images reveals time variations of heart wall motion, thickness, and enclosed area, which remain important for the diagnosis of cardiac disorders. To evaluate these parameters the boundaries of the heart wall must be determined in a reliable fashion.

Computers have been previously used to train operators in tracing heart walls, but the problem of accurate automatic heart wall detection remains. However, the majority of existing algorithms demand at least some input from an operator. Typical examples of required operator interactions are: drawing the initial boundary estimates, specifying the center of the radial search for boundaries [1], placing a cursor within a desired cavity. The detection results often depend on an operator's judgement. In addition, manual initialization remains a tedious and time consuming task.

In this paper, heart wall boundaries are determined by efficient edge detection. To deal with the typical poor quality of echocardiographic images we use a wavelet-based edge detector which can be viewed as smoothing the signal by various degrees and then computing its derivative [3]. We extend the edge detection to three dimensions to include temporal information as an additional degree of freedom.

Our algorithm is fully automatic - it requires no input from a cardiologist. Similar to the method described in [2] the epicardial boundary along the posterior wall is found and used as a reference for subsequent searches in 2-D short-axis echocardiographic images.

This paper is organized as follows: in Section 2 the 3 -D discrete dyadic wavelet transform is presented. Section 3 describes our edge detection scheme. Section 4 describes how boundaries are found from previously detected edges in scale space. Next, Section 5 presents results on both real ultrasound images and on a mathematical phantom. Finally, Section 6 presents our conclusions.

\section{3-D DYADIC WAVELET TRANSFORM}

The wavelet transform can be seen as decomposing a signal onto a set of basis functions which are dilated and shifted versions of a mother function called a wavelet. The discrete wavelet transform can be implemented as a perfect reconstruction filter bank-the transform coefficients are obtained by successive applications of discrete filters. A more detailed treatment of the wavelet transform is presented in [3].

Due to its non-redundant representation, the discrete orthogonal wavelet transform has been particularly successful in image compression. There are, however, tasks in image processing such as edge detection and denoising for which overcomplete (redundant) de- 
compositions may be more useful. The dyadic wavelet transform used in this paper has the advantage of such overcomplete representations (loose frames).

Changing the scale parameter in a dyadic manner (scale $2^{j}$ with $1 \leq j \leq J$ ) allows fast implementation of the discrete dyadic wavelet transform. The following pseudo-code describes the discrete dyadic wavelet transform of an original signal $s_{1}$ :

begin

$$
\begin{aligned}
& j:=0 \\
& \text { while }(j<J)
\end{aligned}
$$

begin

$$
\begin{aligned}
& d_{2^{j+1}}:=s_{2^{j}} * g_{j} \\
& s_{2^{j+1}}:=s_{2^{j}} * h_{j} \\
& j:=j+1
\end{aligned}
$$

end

\section{end}

where $d_{2^{j+1}}$ denotes the wavelet coefficients at a scale $2^{j+1}, g_{j}$ and $h_{j}$ are obtained by inserting $2^{j}-1$ zeros between each of the coefficients of the original filters $g$ and $h$, and the asterisk stands for convolution. The complexity of the above algorithm is $O(N \log N)$, ( $N$ being the number of nonzero samples in $s_{1}$ ). This makes the algorithm suitable for real time image processing requirements.

Edge detection performance puts additional constraints on the wavelet and its filters. To achieve good edge detection results under noisy conditions a signal is smoothed before its derivative is computed[5]. In [3] this idea is applied in a multiscale context. The wavelet transform with the wavelet defined as the derivative of a smoothing function at a certain scale is proportional to the derivative of an original signal smoothed at the same scale. The local extrema of such a wavelet transform corresponds to the inflection points of the smoothed signal. The sharp variation points of the signal smoothed at different scales can be, therefore, detected by finding the local maxima of the wavelet transform magnitude.

A wavelet equal to the first order derivative of a smoothing function must have a zero of order 1 in $\omega=$ 0 . This implies that the filter $G(\omega)$ must have an order 1 zero at $\omega=0$. Additional constraints on filters are placed by a choice of a wavelet that is antisymmetric, as regular as possible, and has compact support.

In [3], two-dimensional wavelets are constructed from one-dimensional discrete dyadic wavelets. Two-dimensional wavelets are simply separable products of functions of the $x$ and $y$ variables. We can extend the 2-D discrete dyadic wavelet transform to three dimensions in a simple manner, where 3 - $D$ wavelets are separable products of functions of the $x, y$, and $z$ variables.
An algorithm for computing the 3 -D discrete dyadic wavelet analysis of a signal $s_{1}$ is shown in the pseudocode below:

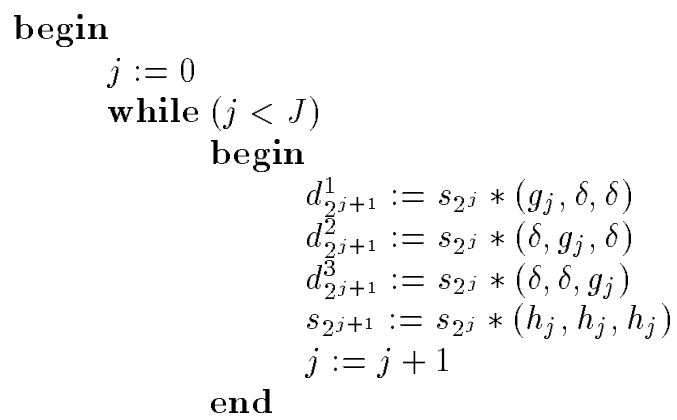

\section{end}

where $d_{2 j+1}^{1}, d_{2 j+1}^{2}$, and $d_{2^{j+1}}^{3}$ denote the wavelet coefficients at a scale $2^{j+1}, g_{j}$ and $h_{j}$ are obtained by inserting $2^{j}-1$ zeros between each of the coefficients of the original filters $g$ and $h, \delta$ is the unit impulse, and the asterisk stands for convolution.

Figure 1 displays the magnitude frequency responses of the three $3-\mathrm{D}$ wavelet filters.

\section{3-D EDGE DETECTION}

To make use of temporal (interframe) and spatial (intraframe) information we treat a sequence of images as a 3-D structure. Two axis refer to the customary spatial coordinates while the third one points in the temporal direction. Edges in the current frame are therefore the result of both information in the current frame and contributions from its neighboring frames.

As previously mentioned in Section 2 the local maxima of the wavelet transform magnitude reveal the sharp variation points of a smoothed signal. To detect the 3-D edges we extend to three dimensions the 2-D procedure previously applied in [6].

Once the 3-D dyadic wavelet transform is computed, the magnitudes and directions of the gradient vectors are determined. At each scale $2^{j}$, the magnitude of the gradient vector at the point $(x, y, z)$ is given by

$M_{2^{j}}(x, y, z)=$

$\sqrt{\left|d_{2^{j}}^{1}(x, y, z)\right|^{2}+\left|d_{2^{j}}^{2}(x, y, z)\right|^{2}+\left|d_{2^{j}}^{3}(x, y, z)\right|^{2}}$,

the angle along the $x$-direction is

$$
\varphi_{2^{j}}(x, y, z)=\operatorname{argument}\left(d_{2^{j}}^{1}(x, y, z)+i d_{2^{j}}^{2}(x, y, z)\right),
$$

and the angle along the $z$-direction equals

$\vartheta_{2^{j}}(x, y, z)=\operatorname{argument}\left(d_{2^{j}}^{3}(x, y, z)+\right.$

$$
\left.i \sqrt{\left|d_{2^{j}}^{1}(x, y, z)\right|^{2}+\left|d_{2^{j}}^{2}(x, y, z)\right|^{2}}\right),
$$



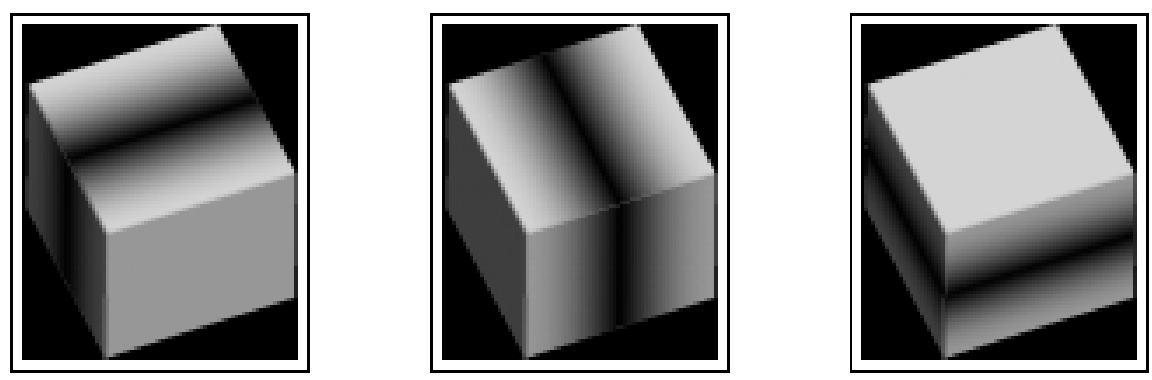

Figure 1: 3-D filters used for multiscale edge detection.

where $d_{2^{j}}^{1}(x, y, z), d_{2^{j}}^{2}(x, y, z)$, and $d_{2^{j}}^{3}(x, y, z)$ denote the wavelet coefficients at the point $(x, y, z)$ and scale $2^{j}$.

A point $(x, y, z)$ is declared as an edge point at location $(x, y, z)$ and scale $2^{j}$ if the magnitude $M_{2^{j}}(x, y, z)$ has a local maximum in the direction of the gradient. Rather than comparing the magnitude $M_{2^{j}}(x, y, z)$

with the two magnitudes at the neighboring locations closest to the gradient direction we interpolate the two values. This is done by constructing a $3 \times 3 \times 3$ cube around each point $(x, y, z)$ and computing two points of intersection between the gradient direction and the cube. Next, the two "new magnitudes" of the wavelet transform are computed for the two intersection points using bilinear interpolation among four vertices located on each of the intersected sides. Finally, the magnitude $M_{2}{ }^{j}(x, y, z)$ is compared to the two interpolated values and identified as an edge if it is greater than the two interpolated values and some threshold.

After the edges are detected we group together neighboring maximum magnitudes. The obtained maxima curves are then thresholded with respect to their length. Figure 2 shows the 2-D echocardiographic image and the detected edges at scales $2^{3}$ and $2^{4}$.

\section{BOUNDARY DETECTION}

The detected edges together with knowledge of cardiac structure and the image acquisition process serve as a basis for the boundary detection.

Information from neighboring frames was incorporated into the current frame using a $3-\mathrm{D}$ dyadic wavelet transform (Section 2) and 3-D edge detection (Section 3). Next, boundary detection is performed using 2-D information of the current frame alone.

Since our algorithm requires no input from an operator, it must automatically determine the starting point for the search of boundaries along the posterior and anterior walls in 2-D short-axis echocardiographic image sequences. In [2] the epicardial border along the posterior wall was the most prominent feature. When the ultrasound wave meets the boundary between two tissues, a portion of the ultrasound wave is reflected while the remainder keeps propagating past the boundary. For specular reflection to occur, it is necessary that the boundary surface be larger than the wavelength of the incident signal. A very high proportion of the incident energy is reflected from the interface between the posterior lung and myocardium-pericardium interface. This is due to their different characteristic impedances, and the fact that this boundary is virtually orthogonal to the signal.

Thus, our algorithm first searches for the epicardial boundary along the posterior wall. The lower part of the image with detected edges is scanned for the first long curve, which is concave up. After the curve is found, its lowest point is determined (if a lowest line is found, its center point is taken).

Having detected the epicardial boundary along the posterior wall, the search for the endocardial boundaries is performed next. We make use of the fact that the cavity tends to be of lower gray-level intensity than the surrounding muscles since there is no tissue to reflect the ultrasound wave inside the cavity. Endocardial boundaries are chosen only among those edges whose wavelet coefficients show a positive derivative in outward directions from the cavity. Since the endocardial boundaries along the posterior and anterior walls are to be detected, we place restrictions on the vertical component of the 3 -D dyadic wavelet transform: positive for search along the posterior wall and negative along the anterior wall. After the endocardial boundaries are detected, we use edges along the anterior wall to find the remaining epicardial boundary.

\section{RESULTS}

Determining the accuracy of the detected boundaries is a difficult task. Since it is impossible to measure the heart wall boundaries "in vivo," we created a mathematical phantom to assist us with validation of our method. The mathematical phantom in Figure 3 is a 
part of a sequence of images that mimics contractions of the heart. After applying our algorithm to the phantom we compared the detected edges to characteristics of the phantom, whose features were known "a priori." Our algorithm was able to reliably localize the edges in these phantom sequences.

Figure 3 shows the mathematical phantom and the detected boundaries. In Figure 4, one image frame from a 2-D short-axis echocardiographic image sequence and the resulting boundaries are presented.

\section{CONCLUSION}

The described edge detection algorithm makes use of both spatial and temporal information in image sequences in a compact manner. Temporal features were not dealt with separately, rather they formed an integral part of the decision process.

The multiscale nature of the edge detection algorithm makes it robust enough for relatively poor image quality typical in 2-D echocardiography. The detected edges promise reliability for edge-based fully automatic detection of epicardial and endocardial boundaries along the posterior and anterior walls in 2-D shortaxis echocardiographic image sequences.

\section{REFERENCES}

[1] C. H. Chu, E. J. Delp, and A. J. Buda, "Detecting left ventricular endocardial and epicardial boundaries by digital two-dimensional echocardiography," IEEE Trans. Med. Imaging, vol. MI-7, pp. 81-90, 1988.

[2] D. C. Wilson, E. A. Geiser, and J.-H. Li, "Feature extraction in two-dimensional short-axis echocardiographic images," J. Math. Imaging Vision, vol. 3, pp. 285-298, 1993 .

[3] S. Mallat and S. Zhong, "Characterization of signals from multiscale edges," IEEE Trans. Pattern Anal. Machine Intell., vol. PAMI-14, pp. 710-732, 1992.

[4] I. Daubechies, Ten Lectures on Wavelets, Society for Industrial and Applied Mathematics, Philadelphia, PA, 1992.

[5] J. Canny, "A computational approach to edge detection," IEEE Trans. Pattern Anal. Machine Intell., vol. PAMI-8, pp. 679-698, 1986.

[6] A. Laine, S. Schuler, and J. Fan, "Multiscale contrast enhancement for digital mammography," IEEE Trans. Med. Imaging, vol. MI-13, 1994. In press.
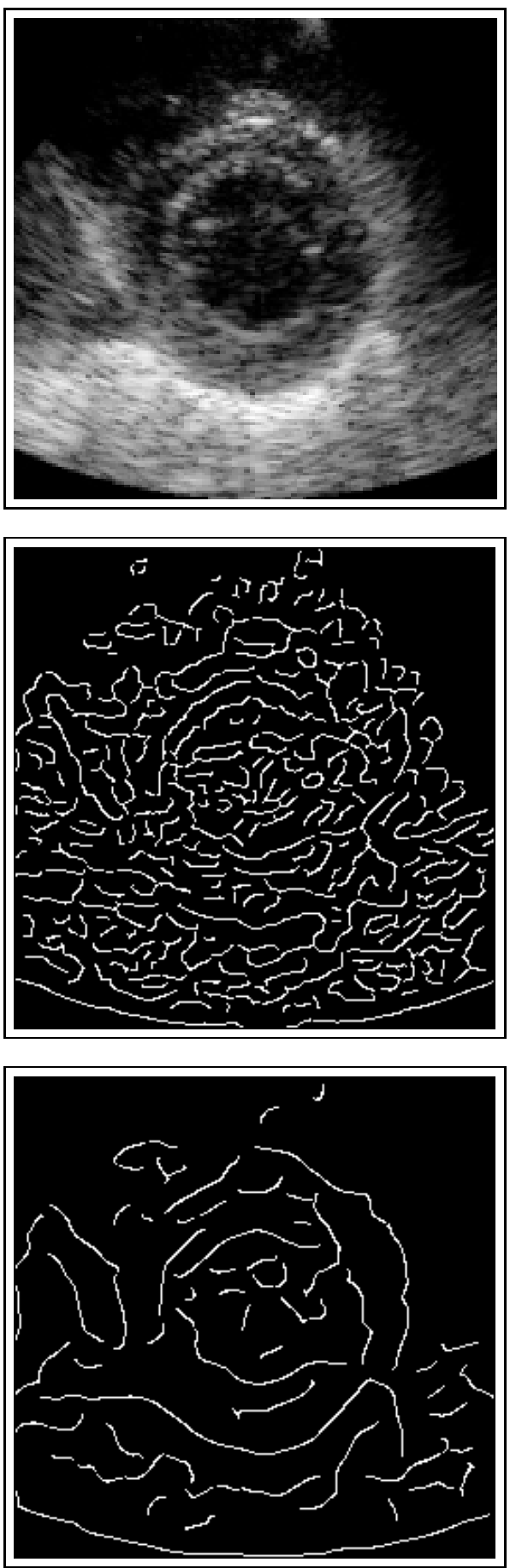

Figure 2: Original image (top) and detected edges at scales $2^{3}$ (middle) and $2^{4}$ (bottom). 

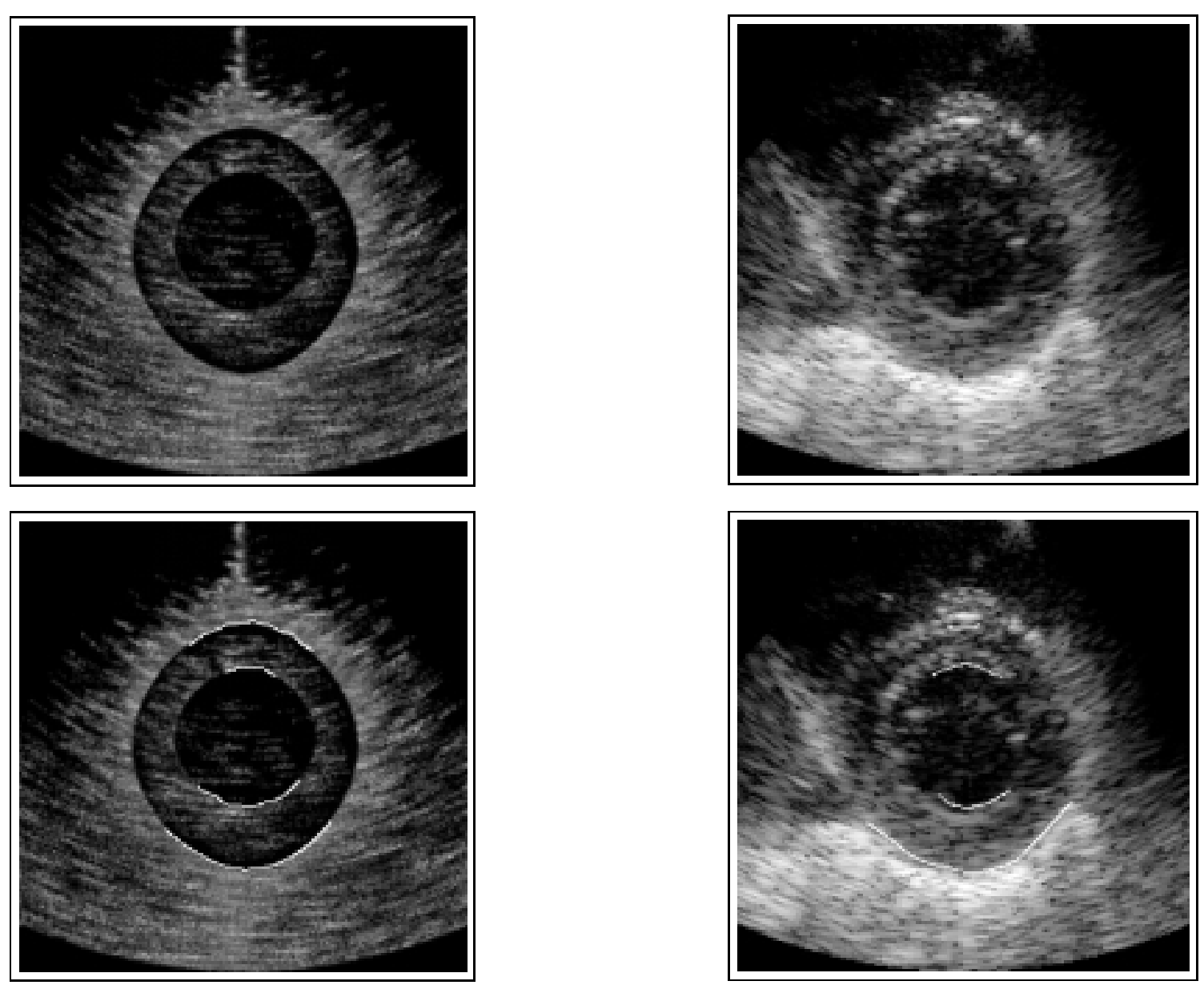

Figure 3: Mathematical phantom (top) and mathematical phantom with detected boundaries superimposed (bottom).
Figure 4: Original image (top) and original image with detected boundaries superimposed (bottom). 